Submitted to The Astrophysical Journal

\title{
Polarization Signatures of Relativistic Magnetohydrodynamic Shocks in the Blazar Emission Region - I. Force-free Helical Magnetic Fields
}

\author{
Haocheng Zhang ${ }^{1,2}$, Wei Deng ${ }^{3,2}, \mathrm{Hui}^{2}{ }^{2}$ and Markus Böttcher ${ }^{4}$
}

\begin{abstract}
The optical radiation and polarization signatures in blazars are known to be highly variable during flaring activities. It is frequently argued that shocks are the main driver of the flaring events. However, the spectral variability modelings generally lack detailed considerations of the self-consistent magnetic field evolution modeling, thus so far the associated optical polarization signatures are poorly understood. We present the first simultaneous modeling of the optical radiation and polarization signatures based on 3D magnetohydrodynamic simulations of relativistic shocks in the blazar emission environment, with the simplest physical assumptions. By comparing the results with observations, we find that shocks in a weakly magnetized environment will largely lead to significant changes in the optical polarization signatures, which are seldom seen in observations. Hence an emission region with relatively strong magnetization is preferred. In such an environment, slow shocks may produce minor flares with either erratic polarization fluctuations or considerable polarization variations, depending on the parameters; fast shocks can produce major flares with smooth PA rotations. In addition, the magnetic fields in both cases are observed to actively revert to the original topology after the shocks. All these features are consistent with observations. Future observations of the radiation and polarization signatures will further constrain the flaring mechanism and the blazar emission environment.
\end{abstract}

Subject headings: galaxies: active — galaxies: jets — gamma-rays: galaxies radiation mechanisms: non-thermal — relativistic processes — polarization

\footnotetext{
${ }^{1}$ Astrophysical Institute, Department of Physics and Astronomy, Ohio University, Athens, OH 45701, USA

${ }^{2}$ Theoretical Division, Los Alamos National Laboratory, Los Alamos, NM 87545, USA

${ }^{3}$ Department of Physics and Astronomy, University of Nevada Las Vegas, Las Vegas, NV 89154, USA

${ }^{4}$ Centre for Space Research, North-West University, Potchefstroom, 2520, South Africa
} 


\section{Introduction}

Blazars are the most violent active galactic nuclei. They are known to emit nonthermaldominated radiation from radio to $\gamma$-rays with strong variability across the entire electromagnetic spectrum. It is generally agreed that the emission comes from an unresolved region in a relativistic jet that is directed close to our line of sight (LOS). The blazar spectrum has two components. The low-energy component, from radio to optical/UV, is known to be polarized, with the polarization percentage ranging from a few to tens of percent. This is in agreement with the synchrotron emission from nonthermal electrons in a partially ordered magnetic field. Several papers have demonstrated that the observed polarization signatures may indicate a helical magnetic structure (Lyutikov et al. 2005; Pushkarev et al. 2005; Zhang et al. 2015). The high-energy component, from X-rays to $\gamma$-rays, is usually interpreted as originating from inverse Compton scattering by the same nonthermal electrons of soft seed photons (e.g., Marscher \& Gear 1985; Dermer et al. 1992; Sikora et al. 1994), but a hadronic origin cannot be ruled out (e.g., Mannheim \& Biermann 1992; Mücke \& Protheroe 2001; Böttcher et al. 2013). Both spectral components of blazars exhibit fast variability. Many observations show flares in various observational bands lasting days or even hours (e.g., Ciprini 2011; Chatterjee et al. 2012); in particular, high-energy $\gamma$-rays sometimes show variability time scale within several tens of minutes (e.g., Aharonian et al. 2007; Albert et al. 2007). Based on the causality relation, $R \lesssim \delta c t$, where $\delta$ is the Doppler factor and $t$ is the observed flaring time scale, the size of the emission region $R$ in the comoving frame of the emission region should generally be within $0.1 \mathrm{pc}$. Therefore, it is often argued in the blazar spectral variability fittings that the blazar emission region is a small region near the broad line region of the blazar jet (e.g., Tavecchio et al. 2010; Böttcher et al. 2013; Barnacka et al. 2014).

Blazar spectral fittings have shown that the nonthermal electron spectra responsible for the common blazar SEDs require power-law indices of $\gtrsim 2$ in most cases (e.g., Böttcher et al. 2013). This is in agreement with numerical simulations of relativistic shocks, which have demonstrated that diffusive shock acceleration forms such power-law spectra (Achterberg et al. 2001; Spitkovsky 2008; Summerlin \& Baring 2012). Therefore, shocks are frequently used to explain blazar flaring activities. However, in general, numerical simulations of shock acceleration do not provide detailed calculations of the expected radiation features. In terms of the spectral variability fittings, scenarios of relativistic shocks propagating through the jet have been widely investigated (e.g., Marscher \& Gear 1985; Spada et al. 2001; Joshi \& Böttcher 2007; Graff et al. 2008). These models have successfully fit the timedependent SEDs and multiwavelength light curves of blazar flares, but they usually assume a stationary chaotic magnetic field. Therefore, the polarization variations are poorly understood and/or constrained. 
Polarimetry plays an important role in constraining the jet physics. Radio polarimetry has been a standard tool to understand magnetic fields of large-scale jets in radio galaxies (e.g., Laing \& Bridle 2014). Additionally, sometimes blazar flaring activities have been reported to be connected with changes in radio knots (e.g., Marscher et al. 2008). However, since blazar emission regions remain spatially unresolved, high-resolution imaging and polarimetry at radio wavelengths usually cannot directly constrain the physical conditions in the blazar high-energy emission environment. However, the optical emission from the blazar emission region generally dominates over all the other optical emissions from the blazar jet, hence optical polarimetry is able to directly reveal the inner-jet magnetic field structure. The observed optical polarization signatures are generally erratic. Typically, the polarization degree $(\mathrm{PD})$ varies within $\sim 30 \%$, but occasionally a higher PD $(\sim 40 \%)$ is reported (e.g., Scarpa \& Falomo 1997). The polarization angle (PA) usually displays perturbations around some mean values. However, polarization signatures can be highly variable during the flaring activities. In particular, significant multiwavelength flaring activities are seen to be accompanied by large $\left(\gtrsim 180^{\circ}\right)$ PA rotations (e.g., Marscher et al. 2008; Abdo et al. 2010; Chandra et al. 2015). These flare + PA rotation events often feature smooth PA rotations and apparently time-symmetric light curves and PD patterns. In addition to the individual observations, Blinov et al. (2015) are performing a polarization monitoring program of a large sample, in which they find that blazars with detected PA rotations generally exhibit stronger variations in the PA, and the rotations can go in both directions. Moreover, they claim that the PA rotations are probably physically connected to the $\gamma$-ray flares.

In terms of modeling, several mechanisms have been put forward to understand the polarization signatures, especially the PA rotations. An emission region following a helical trajectory (e.g., Marscher et al. 2008) can give rise to a smooth PA rotation, but such a model prefers all rotations present in the same blazar to follow the same rotating direction, which contradicts observations. An initially chaotic magnetic field structure compressed by a flat shock (Laing 1980) or multiple small shocks (Marscher 2014) can produce erratic polarization patterns and occasionally large polarization variations through random walks, but the resulting patterns are normally whimsical, and it has been shown that the observed PA rotations are unlikely to result completely from random walks (Kiehlmann et al. 2013; Blinov et al. 2015). Chen et al. (2014) and Zhang et al. (2014) have proposed a model in which a disturbance propagates through a cylindrical emission region pervaded by a helical magnetic field. This model can give rise to systematic PA rotations and apparently time-symmetric light curves and PD patterns. Based on this model, Zhang et al. (2015) and Chandra et al. (2015) have presented simultaneous fittings of SEDs, light curves and polarization signatures for two flare + PA rotation events. Nonetheless, in all the models mentioned above, the magnetic field evolution is treated in an ad hoc way. Furthermore, 
none of them can so far explain the statistical properties of the polarization variations as shown in Blinov et al. (2015).

In this paper, we present the first polarization-dependent radiation modeling based on relativistic magnetohydrodynamics (RMHD) simulations of shocks in helical magnetic fields in the blazar emission region. This new approach performs detailed analysis on the interaction between the shock propagation and the magnetic field evolution, which enables us to constrain the blazar emission environment and the shock parameters. We demonstrate that the blazar emission region largely possesses significant magnetic energy. In such an environment, fast shocks can be the driver of flaring events, producing both the typical polarization fluctuations and the occasional large PA rotations. Our simulation results are

consistent with the statistical polarization properties reported in Blinov et al. (2015). We will describe our model setup in Section 2, illustrate the interaction between the shock and the magnetic field as well as the consequent radiation and polarization signatures in Section 3, present additional parameter studies in Section 4, and discuss the results in Section 5.

\section{Model Setup}

The purpose of this paper is to study the time-dependent radiation and polarization signatures resulting from an RMHD shock in the blazar emission environment, with the simplest physical assumptions. In this section, we will describe our physical assumptions and corresponding code structure in detail.

\subsection{Physical Assumptions}

The blazar emission region is often considered to be an unresolved region near the broad line region of the jet. Generally speaking, the magnetic field topology and evolution as well as plasma flow dynamics in the blazar emission region should be linked with those in the largescale jet. However, the oberved fast variability and high luminosity suggest that the emission region is an extraordinary, very small and localized region with fast evolution. Therefore, we argue that within the time scale of an individual flare, the blazar emission region can be considered as uncorrelated with the properties of the large-scale jet, even though it is spatially embedded within the jet. On the other hand, the emission region is expected to remain well localized in the jet during flares due to pressure provided by the large-scale jet structure. We assume that flares are due to a disturbance propagating through the emission region. Many observations show that light curves and even the time-dependent polarization 
signatures appear symmetric in time (e.g., Abdo et al. 2010). This means that the emission region and the disturbance are likely to possess some kind of symmetry. Zhang et al. (2014, 2015) have shown that a helically symmetric emission region and disturbance, with detailed consideration of light-travel-time effects (LTTEs), can naturally explain the apparently timesymmetric light curves and time-dependent polarization signatures. Therefore, we continue to use that assumption here.

Due to the relativistic aberration, even though we are observing blazars nearly along the jet in the observer's frame (typically, $\theta_{o b s, 1} \sim 1 / \Gamma_{1}$ ), where $\theta_{o b s, 1}$ and $\Gamma_{1}$ are the angle between the LOS and the jet direction and the Lorentz factor of the emission region in the observer's frame, respectively, the angle $\theta_{o b s}$ between LOS and the jet axis in the comoving frame is likely around $90^{\circ}$ (if $\theta_{o b s, 1} \sim 1 / \Gamma_{1}$, then $\theta_{o b s} \sim 90^{\circ}$ ). Zhang et al. (2014) have shown that if the LOS is fixed at some other angles, the general trend of polarization variations is only weakly affected. In addition, as is suggested in the bending jet scenario of the polarization signatures, a change in the LOS direction may significantly affect the polarization signatures. However, those effects are beyond the scope of our first-step study. Thus in all of the following simulations, we choose $\theta_{o b s}=90^{\circ}$ in the comoving frame, and hence the Doppler factor $\delta \equiv\left(\Gamma_{1}\left[1-\beta_{\Gamma_{1}} \cos \theta_{o b s, 1}\right]\right)^{-1} \sim \Gamma_{1}$.

We use ideal RMHD simulations to describe the evolution of the system. The underlying model assumes that a plasma jet is traveling with a Lorentz factor of $\Gamma_{0}$ in the observer's frame. In the comoving frame of the jet, a flow of plasma which is pervaded by a helical magnetic field travels at a Lorentz factor of $\Gamma$. Inside this flow lies a cylindrical emission region containing nonthermal particles. Along the path of the flow it encounters a flat stationary layer of the plasma (the disturbance), forming a shock wave. Thus the total Lorentz factor of the emission region in the observer's frame is $\Gamma_{1}=\Gamma \times \Gamma_{0}$. Although in the following sections our RMHD simulations use different Lorentz factors $\Gamma$ of the disturbance in the comoving frame of the emission region, $\Gamma_{1}$ of the emission region in the observer's frame is not constrained. Therefore, we assume a fixed Lorentz factor of $\Gamma_{1}=20$ for the emission region in the observer's frame, which is commonly inferred from SED modelings.

Before the emission region interacts with the disturbance, the helical magnetic field is assumed to be in force-balance. The advantage of this magnetic field setup is that it will naturally give rise to comparable poloidal and toroidal contributions, resulting in a relatively low background PD without the need of any turbulence. In addition, this can simplify our setup for the plasma density and the thermal pressure, which are taken to be uniform inside the emission region. In this way, we assume that initially all flow conditions and magnetic fields are laminar, with no preexisting turbulent components. Also, as the RMHD requires an input for the thermal pressure, we assume that in the beginning the plasma is cold and 
hence the thermal pressure is very small compared to the kinetic energy or the magnetic energy.

In the comoving frame of the emission region, the disturbance and the resulting shock will propagate through the emission region, and change the physical conditions as well as accelerate particles at its location, generating a flare. We apply the simple assumption that the shock will inject fresh nonthermal electrons at the shock front whose energy is equal to a fixed small amount of the local shock kinetic energy. In this way, any deceleration and deformation of the shock due to the magnetic field obstruction can be taken into consideration. In Section 4 we will show that the injection rate does not affect the polarization signatures too much.

We briefly summarize our model assumptions in the following:

1. The emission region is a small cylindrical region embedded in the large-scale jet.

2. The evolution of the emission region is detached from the large-scale jet.

3. The boundary of the emission region is held by a pressure wall, probably provided by the large-scale jet.

4. In the comoving frame of the emission region, the observer is observing from the side of the emission region $\left(\theta_{\mathrm{obs}}=90^{\circ}\right)$.

5. The initial magnetic field inside the emission region is a force-free helical magnetic field.

6. Initially all flow conditions and magnetic fields are laminar.

7. Initially the plasma is cold.

8. Initially the disturbance is a flat cylindrical region traveling relativistically in the comoving frame of the emission region.

9. The disturbance will generate a shock in the emission region. At the shock front, the shock will inject nonthermal particles whose total energy is a constant, small fraction of the local shock kinetic energy.

\subsection{Code Structure}

The above model is realized by the combination of the 3D multi-zone RMHD code LA-COMPASS developed by Li \& Li (2003) and the 3D multi-zone polarization-dependent ray-tracing code 3DPol developed by Zhang et al. (2014). Fig. 1 (left) shows a sketch of the simulation setup, and Table 1 presents some major parameters. The RMHD simulation is performed in the comoving frame of the emission region, using Cartesian coordinates. We apply the outflow boundary conditions for the simulation domain; in the following, we will demonstrate that our setup should not suffer from any boundary effects. The simulation domain is pervaded by a helical magnetic field (Fig. 1 (left)). In reality, the poloidal 
magnetic field lines have to be closed, but based on our assumption the returning magnetic flux is generally outside the simulation domain. The helical magnetic field is assumed to be in force-balance, in the form of

$$
\begin{aligned}
& B_{z}=B_{0} \times J_{0}(k r) \\
& B_{\phi}=B_{0} \times J_{1}(k r)
\end{aligned}
$$

where $J_{0}$ and $J_{1}$ are the Bessel functions of the first kind, $r$ is the radius, and $B_{0}$ and $k$ are normalization factors of the magnetic field strength and the radius, respectively. The $B_{r}$ component is assumed to be zero. In order to avoid any boundary effects in $x, y$ directions and comply with the assumed generally monodirectional $B_{z}$, we add an exponential cutoff for both components when $B_{z}$ approaches zero $(k r \sim 2.4)$. However, the exponential cutoff will lead to a nontrivial magnetic force at the edge of the simulation domain, hence we add a plasma pressure to balance it (Fig. 11 (left)).

\begin{tabular}{|l|c|c|c|}
\hline Parameters & Length & Time & Velocity \\
\hline Relation & $L_{0}$ & $L_{0} / c$ & $c$ \\
\hline Code Unit & 1 & 1 & 1 \\
\hline Physical Value & $8.33 \times 10^{16} \mathrm{~cm}$ & $2.78 \times 10^{6} \mathrm{~s}$ & $3 \times 10^{10} \mathrm{~cm} \mathrm{~s}^{-1}$ \\
\hline Parameters & Magnetic Field & Thermal Pressure & Plasma Density \\
\hline Relation & $B_{0}$ & $B_{0}^{2} /(4 \pi)$ & $B_{0}^{2} /\left(4 \pi c^{2}\right)$ \\
\hline Code Unit & 1 & 1 & 1 \\
\hline Physical Value & $0.1 G$ & $8.0 \times 10^{-4} \mathrm{erg} \mathrm{cm}^{-3}$ & $8.8 \times 10^{-25} \mathrm{~g} \mathrm{~cm}^{-3}$ \\
\hline
\end{tabular}

\begin{tabular}{|l|c|}
\hline Bulk Lorentz factor $\Gamma_{1}$ & 20 \\
\hline Orientation of LOS $\theta_{\text {obs }}\left({ }^{\circ}\right)$ & 90 \\
\hline Electron minimal energy $\gamma_{1}$ & $10^{3}$ \\
\hline Electron maximal energy $\gamma_{2}$ & $5 \times 10^{4}$ \\
\hline Electron power-law index $p$ & 2 \\
\hline
\end{tabular}

Table 1: Summary of parameters. Top: Conversion between the RMHD code units and the physical value. Bottom: Additional parameters used in the 3DPol simulation. All parameters in this table are in the comoving frame of the emission region, except the bulk Lorentz factor.

The emission region is set to be a fixed volume in the simulation domain $(r \sim 2.5$ and $z$ ranges from -2.5 to 2.5 , see Fig. 2). Since the simulation domain is in the comoving frame of the emission region, the disturbance will travel at a Lorentz factor of $\Gamma$ in the $z$ direction. We assume that the disturbance is a thin layer ( 0.5 in width) and initially put it at some distance away from the emission region (the disturbance layer ranges from -8 to -7.5 ) to allow some time for the shock wave to form in the numerical simulation (see Fig. 1 (left) and Fig. 2). In order to avoid boundary effects in $z$ direction, we use a sufficiently large range in $z$ ( -20 to 20$)$, so that at the end of the simulation, no signal has reached the $z$ boundary. We define a magnetization factor in the emission region,

$$
\sigma=\frac{E_{e m}}{h}
$$


where $E_{e m}=\frac{B^{2}+E^{2}}{8 \pi}$ is the electromagnetic energy density, $h=\rho c^{2}+\frac{\hat{\gamma} p}{\hat{\gamma}-1}$ is the specific enthalpy, $\rho$ is the plasma density, $\hat{\gamma}$ is the adiabatic index and $p$ is the thermal pressure. We further assume that the plasma is cold. Hence we choose $\frac{\hat{\gamma} p}{\hat{\gamma}-1}$ to be a small part (1/30) of the electromagnetic energy initially. In this way the magnetization factor is approximately $\sigma \sim \frac{E_{e m}}{\rho c^{2}}$. The relativistic Alfvén speed can then be expressed as

$$
V_{A} \sim \frac{c}{\sqrt{4 \pi \rho c^{2} / B^{2}+1}}
$$

Since the entire simulation domain is axisymmetric, we present a cut in the $x z$ plane in Fig. 2 to illustrate the initial condition, including the magnetic field and the velocity of the disturbance. Notice that the $B_{x}$ component is not shown, as in a helical setup, in the $x z$ plane this component is trivial. Also this figure is for an initial $\Gamma=10$; for other $\Gamma$ the velocity appears slightly different. As the RMHD simulation takes Cartesian coordinates, we will transform the results to the cylindrical coordinates and feed into the 3DPol code.

The 3DPol code is focused on the polarization-dependent synchrotron radiation. It uses a cylindrical geometry, and further divides it evenly into multiple zones in $r, \phi$ and $z$ directions. Each zone has its own magnetic field evolution, which is provided by the RMHD simulations. The nonthermal electrons in each zone are assumed to range between a minimal and a maximal Lorentz factor $\gamma_{1}$ and $\gamma_{2}$, with a fixed spectrum of power-law index of -2 plus an exponential cutoff at the high-energy end,

$$
n(\gamma)=n \gamma^{-2} e^{-\frac{\gamma}{\gamma_{2}}}, \text { for } \gamma>\gamma_{1}
$$

The nonthermal electron normalization factor $n$ is assumed to have two components: a fixed uniform initial background density $n_{b}$, and an injected density $n_{i}$ during the shock. As the electron spectrum is fixed, we will only consider the optical light curves and polarization patterns, which do not suffer from any synchrotron-self absorption and Faraday rotation effects for our parameters. As in the RMHD simulation, all calculations are performed in the comoving frame of the emission region. Given the local magnetic field information and the nonthermal electron population, the 3DPol code will calculate the Stokes parameters at various frequencies at every time step in each zone. Next the code will employ ray-tracing to take account of all LTTEs (see Fig. 1 (right) for an illustration), and add up the incoherent Stokes parameters arriving at the observer at the same time, then Lorentz transform to the observer's frame so as to obtain the total time-dependent radiation and polarization signatures.

We define the PA in the following way. When the electric vector is parallel to the emission region propagation, $P A=0$ (toroidal component dominating). Since the PA has $180^{\circ}$ ambiguity, the toroidal dominance happens at $P A=2 N \times 90^{\circ}$, and the poloidal dominance at $P A=(2 N+1) \times 90^{\circ}$, where $N$ is an integer. 


\section{Interaction between Shock and Magnetic Field}

In this section, we will investigate how the shock speed and the magnetization in the blazar emission environment will affect the time-dependent radiation and polarization signatures. Since we generate the shock by a relativistic disturbance, and the simulation is done in the comoving frame of the emission region, the shock front and its motion can hardly be distinguished from the disturbance. Moreover, as we will see in the following results, during the propagation, both the disturbance and the shock speeds may vary in time. Hence we

use the initial Lorentz factor $\Gamma$ of the disturbance rather than the actual time-dependent shock speed for the parameter study. For the same reason, the magnetization factor $\sigma^{\prime}$ in the shock frame will vary in time as well; we choose the initial $\sigma$ in the emission region instead. This $\sigma$ is also a direct indicator of how much the emission region is magnetized at the beginning. By comparing the results with the general observational features, we will be able to constrain the physics in the emission environment. For this purpose, we will present three cases, namely, $\sigma \sim 0.01, \Gamma=10$ (Case I), $\sigma \sim 1, \Gamma=10$ (Case II) and $\sigma \sim 1, \Gamma=3$ (Case III). The RMHD simulation results are shown in Figs. 3 , 5 and 6 for Case I, II and III, respectively, along with the associated radiation and polarization signatures in Fig. 7. For all the cases studied, we fix the initial nonthermal electron density $n_{b}$ and the characteristic magnetic field strength $B_{0}$. The magnetization factor is adjusted through changing the plasma density. Since we employ a simple electron spectrum, we only study the light curves and polarization patterns in the optical band as examples. Additionally, as the background nonthermal electron density and the injection rate are set as input parameters, we use the relative flux level, where 1 is approximately $10^{-11} \mathrm{erg} \mathrm{cm}^{-2} \mathrm{~s}^{-1}$.

\subsection{Effects of Magnetization}

We first examine a kinetic energy dominated environment, Case I. Such an environment is frequently assumed in a shock-initiated flaring model. We first notice that in a trivially magnetized setting $(\sigma \sim 0.01)$, the speed of Alfvén waves, $V_{A} \sim 0.1 c$, is much slower than that of the shock/disturbance. This speed is even slower inside the disturbance. As a result, the entire simulation domain can be treated similar to a hydro setup. Consequently, we can expect that the shock/disturbance will strongly compress the plasma at the shock front, and push it along with the propagation. However, in the ideal MHD, the plasma and the field lines are coupled (frozen-in field lines). Therefore, the toroidal magnetic field lines at the shock front will be greatly compressed, leading to a much larger toroidal contribution; meanwhile, far downstream of the shock, the toroidal lines will be sufficiently stretched, giving rise to a perfectly ordered poloidal magnetic field (see Fig. 3, $B_{y}$ ). The physical effects described 
above are the consequence of a shock in a nearly hydro environment with the assumption of the frozen-in field lines. Therefore, we expect that the above shock modifications to the magnetic field structure is not limited to our initial magnetic field topology, but will happen in other magnetic field setups as well. Notice that, however, due to the shock compression, the initial force balance in the radial direction between the magnetic field and plasma pressure and density may be broken. This will lead to some hydrodynamical perturbations in the plasma density and pressure in the radial direction, which are explicitly illustrated in Fig. 4. However, those perturbations are only transported at sonic speeds. This is supported by Fig. 4 where any plasma velocities in the radial direction that arise from those perturbations are non-relativistic. Therefore, although given enough time they may significantly modify the post-shock structures, they are much slower than the time scale that we are interested in in our RMHD simulation. Therefore, we find that those hydrodynamical effects are of minor importance to our current study, and will not discuss them in the following.

Case II has a moderately magnetized environment $(\sigma \sim 1)$. Hence, the magnetic field will actively participate in the shock propagation. Komissarov \& Lyutikov (2011) have calculated that the maximal relativistic shock compression ratio in a magnetized environment is given by

$$
r_{c}=\frac{6\left(1+\sigma^{\prime}\right)}{1+2 \sigma^{\prime}+\sqrt{16 \sigma^{\prime 2}+16 \sigma^{\prime}+1}}
$$

where $r_{c}$ and $\sigma^{\prime}$ are the shock compression ratio and the magnetization factor in the shock frame, respectively. As a result, at the shock front, we can see from the simulation that the shock compression becomes weaker due to the higher magnetization (Fig. 5, $B_{y}$ ). Furthermore, although initially the emission region has a negligible magnetic force, the enhancement in the toroidal component at the shock front will break down this balance, resulting in a contracting magnetic force in the $r$ direction, given by

$$
F_{B}=-\frac{\partial}{\partial r} \frac{B_{\phi}^{2}+B_{z}^{2}}{2}-\frac{B_{\phi}^{2}}{r}
$$

This contraction is transported by Alfvén waves, which are mildly relativistic in this case. Therefore, in addition to the compression in the $z$ direction at the shock front, there exists a contraction of the plasma in the $r$ direction. Because of the frozen-in field lines, the poloidal component will be strengthened at the shock front (Fig. 5, $B_{z}$ ). We can see from the simulation that at a later stage, the increase in the poloidal component gradually gets closer to that in the toroidal component, and even the shape of the shock/disturbance is modified, showing a bullet shape at the central part (Fig. $5 V_{z}$ ). Moreover, at variance with Case I, far downstream of the shock/disturbance, even though some plasma is pushed away by the shock/disturbance, the magnetic field is observed acting to revert to its initial topology (Fig. 5. $B_{y}$ and $\left.B_{z}\right)$. 
In the spectral variability fitting, it is frequently argued that during the shock propagation, the local magnetic field either generally maintains its strength and topology (Sokolov et al. 2004; Joshi \& Böttcher 2007), or reverts to its initial state after the shock (Chen et al. 2014; Zhang et al. 2014). This requirement is often necessary to produce the best fittings. Our simulations demonstrate that this can only happen in an adequately magnetized emission region. Therefore, detailed analysis of the magnetic field evolution and shock dynamics should play an essential part in those spectral variability models.

We notice that in both simulations, there are regions with negative $V_{z}$ (Figs. 3 and 5 , $V_{z}$ ). This is likely due to the reverse shock. In reality, the reverse shock may also lead to some radiation and polarization signatures, but its effect is expected to be weaker than the main shock. Thus in the following we will not consider it.

Now we will consider the radiation and polarization signatures resulting from the above RMHD simulations, calculated using the 3DPol code. The general trends are similar for both cases. Before the shock moves in, the entire emission region is axisymmetric. Although the poloidal and toroidal components are comparable in the force-free setup, due to the LOS effect, the projected toroidal component onto the plane of sky is weaker than the projected poloidal component. Therefore initially the emission region has a total polarization dominated by the poloidal component (Fig. 7). When the shock moves in, the toroidal component is strongly increased and fresh nonthermal electrons are injected at the shock front. However, due to the LTTEs, only a small elliptical region on the right that is near the observer is seen (the near side, see Fig. 1 (right), red). Therefore, the flux is seen to gradually increase. Nevertheless, since the toroidal enhancement is strong, a small flaring region is adequate to push the polarization to be dominated by the toroidal component. As a result, the PA quickly rotates to $180^{\circ}$ and forms a plateau, which corresponds to the toroidal domination. The PD first experiences a drop which indicates a switch in the domination between the two components, then climbs up to a high level, implying a strong toroidal dominance (Fig. 7). When the shock is about to leave the emission region, the flaring region reaches maximum (Fig. 1 (right), green). Hence the flux peaks. After that, the flaring region moves far from the observer to the left (the far side, Fig. 1 (right), blue), so the flux gradually decreases in an apparently symmetric pattern. However, as some plasma and the coupled magnetic field lines have been pushed away by the shock/disturbance, the total magnetic field strength is smaller than the initial state. Hence the flux level is lower than the initial value. For the same reason, the toroidal contribution is weakened near the end of the flare, hence the PD rises up higher than the initial value, revealing a stronger poloidal contribution. Since the helical magnetic field direction on the far side is opposite to that on the near side, the PA instead completes a $180^{\circ}$ rotation to the initial value $\left(180^{\circ} \mathrm{PA}\right.$ ambiguity, Fig. (7). 
The major differences between Case I and II are the following. First, due to the stronger magnetic field, Case II has a weaker compression in the toroidal component at the shock front than Case I, while it experiences a rise in the poloidal contribution. Therefore, the PA rotation in Case II appears smoother and has a shorter toroidal dominated plateau than Case I. Also the maximal flare level in Case II is lower. More importantly, near the end of the flare, the moderate magnetization in Case II is acting to restore the initial magnetic topology, hence even if the PD rises above the initial value, it immediately starts to decrease, indicating the recovery of the toroidal component (Fig. 7). Abdo et al. (2010) have shown that at the end of a flare + PA swing event in $3 \mathrm{C} 279$, the PD rises up above the initial value, which is immediately followed by a significant restoration to approximately the initial PD level. The same restoring phases are seen in a number of flare + PA rotation events as

well (Larionov et al. 2008, 2013; Morozova et al. 2014). On the other hand, in Case I the magnetization is too weak, thus the PD rises up to $\sim 70 \%$ at the end of the flare and shows no restoration, indicating a purely poloidal magnetic structure. As is mentioned above, such a phenomenon is generally the consequence of a trivially magnetized environment, and it is expected to happen with other initial magnetic field topologies. Therefore, we argue that such drastic changes in the polarization signatures are unrealistic compared to observations, so that a nearly unmagnetized emission environment model can be ruled out.

\subsection{Effects of Shock Speed}

The effects of the shock speed are rather straightforward. We will illustrate those by examining Case III, which shares the same magnetization as Case II but has a slower disturbance. A slower disturbance has less kinetic energy. Therefore, as the shock/disturbance propagates, it cannot exert a very strong pressure onto the plasma; in return the plasma will decelerate the shock/disturbance. Thus we see in Fig. 6 that the shock/disturbance is considerably slower than the initial value $(\Gamma=3)$. Therefore, the flare duration is much longer than the previous cases (Fig. 7). Also the shock/disturbance will provide less compression in the plasma at the shock front. As a result, we observe in the simulation that the toroidal component is only moderately increased and stretched at the shock front and downstream of the shock/disturbance, respectively (Fig. 6, $B_{y}$ ). Moreover, the shower shock/disturbance takes longer time to propagate through the emission region, allowing for further information exchange by Alfvén waves. Therefore, the contraction of the plasma at the shock front, due to the enhanced toroidal component, is seen to catch up with the shock compression. This leads to a considerably strengthened poloidal component at the shock front. Consequently, the PA rotation disappears; instead it shows fluctuations around the initial value. However, at the flare top, the poloidal contribution is only marginally stronger than the toroidal con- 
tribution. Therefore, the PD still displays a decrease at the middle of the flare (Fig. 7). Meanwhile, downstream of the shock/disturbance, Alfvén waves restore the initial magnetic field topology to a large extent (Fig. 6, $B_{y}$ and $B_{z}$ ). Hence the final PD is not too much higher than the initial value, and gradually recovers after the flare (Fig. 7). Another interesting effect is that due to the weaker kinetic energy in the shock/disturbance and longer influence of Alfvén waves, the shape of the shock/disturbance is distorted (Fig. 6, $V_{z}$ ). Thus the polarization patterns, especially the PA, appear less symmetric in time than the previous cases (Fig. 7).

\section{Parameter Study}

In this section, we will perform parameter studies to constrain the shock speed and the magnetization of the emission environment, by comparing the polarization signatures with the general observational properties. Since the injection rate at the shock front is somewhat arbitrary, we will not compare the light curves. Although in principle the injection rate will affect the polarization signatures, in the following we will demonstrate that the polarization signatures are mainly the consequence of the magnetic field evolution. Here we use moderate $(\Gamma=6)$ and slow $(\Gamma=3)$ speeds, as well as weak $(\sigma \sim 0.1)$, strong $(\sigma \sim 10)$ and moderate $(\sigma \sim 1)$ magnetization factors as examples.

\subsection{Weakly Magnetized Environment, $\sigma \sim 0.1$}

We study a weakly magnetized setup $(\sigma \sim 0.1)$, with the application of moderate $(\Gamma=6)$ and slow $(\Gamma=3)$ disturbances. Due to the small magnetization factor, the mediation by Alfvén waves is slow. Nevertheless, especially in the case of a slow disturbance, the effects of the magnetization cannot be overlooked: the poloidal component is slightly enhanced at the shock front, and the shape of the shock/disturbance is changed (Fig. 8, $B_{z}$ and $V_{z}$ ). As a consequence, the polarization patterns appear relatively smooth, in particular the PA

rotation (Fig. 9). However, downstream of the shock/disturbance, Alfvén waves fail to restore the initial magnetic topology. Hence at the end of the flare, the PD rises up and maintains a high level ( $\sim 40 \%)$, without showing any restoring phase (Fig. 9). This implies that in a weakly magnetized emission environment, shocks can easily alter the magnetic topology, causing significant changes in the polarization signatures. On the contrary, such polarization variations are seldom reported in observations. Therefore, we do not favor a weakly magnetized emission environment. 


\subsection{Strongly Magnetized Environment, $\sigma \sim 10$}

Next we consider an emission region with a high magnetization factor $(\sigma \sim 10)$. Here Alfvén waves become relativistic, thus the shock compression is much weaker. All effects of the magnetization as in Case II and III show up. The difference is that even in the case of a medium speed disturbance $(\Gamma=6)$, the increase in the poloidal component is higher than that in the toroidal field at the shock front (Fig. 10, $B_{y}$ and $B_{z}$ ). Therefore, the PA patterns for both cases exhibit no rotation (Fig. 11). Additionally, the shock/disturbance is greatly distorted. Hence both the PD and the PA patterns appear rather erratic, showing a lot of bumps throughout the flare (Fig. 11), even though the general geometry is rather symmetric. Finally, the restoration downstream of the shock/disturbance is very fast (Fig. $10 B_{y}$ and $B_{z}$ ), thus the PD only rises up gently above the initial value, and quickly recovers at the end of the flare (Fig. 11).

Notice that for a sufficiently fast shock/disturbance, a PA rotation may reappear. However, we expect that such a rotation is likely to be very smooth, without a long toroidal dominated plateau as in Case I to III. Meanwhile the PD variation can be within some small value. This may agree with the observed flare + PA rotation events. To summarize, a strongly magnetized emission environment may generate the typical erratic perturbations in the polarization signatures in the condition of a relatively slow disturbance, together with the smooth flare + PA rotation events provided a relatively fast disturbance. Thus we prefer an emission region with high magnetization.

\subsection{Transition Point: Moderately Magnetized Environment, $\sigma \sim 1$}

Finally we take a look at an intermediate condition, $\Gamma=6$ and $\sigma \sim 1$. This case shares the same magnetization factor as Case II and III. From the simulation we can see that the general features are similar, but the increases in the poloidal and the toroidal components at the shock front are nearly identical (Fig. 12 (left), $B_{y}$ and $B_{z}$ ). However, since the emission region is a cylinder, a larger fraction (at larger radii) of the emission region possesses the enhanced toroidal component. Hence we expect that the radiation during the flare should have more toroidal contributions. In order to test the effects of the injection rate, we perform two 3DPol runs: one with an artificially increased injection rate (approximately ten times higher than the normal value), and one with no injection; the results are shown in Fig. 12 (right). We observe that although the flare level of the high injection case is much higher than the no injection case, the polarization variations appear similar (Fig. 12 (right)). This

indicates that the polarization signatures in our calculation are largely due to the magnetic field evolution, instead of the ad hoc nonthermal electron injection rate. We can see that 
the PA rotation does not show significant toroidal dominated plateau. A number of flare + PA rotation events feature continuous PA rotations without any obvious plateau step (e.g., Marscher et al. 2008; Abdo et al. 2010; Chandra et al. 2015). Based on our simulations, we suggest that such events are likely originating from a sufficiently magnetized environment with a relatively fast disturbance traveling through. Also in this case a vigorous restoring phase is present.

\section{Discussions and Summary}

We have presented the first polarization-dependent radiation modeling based on RMHD simulations of relativistic shocks in helical magnetic fields in the blazar emission region. We find in Section 3 that the magnetic field evolution during the shock is intrinsically governed by a competition between the shock speed and the magnetization in the emission region: the shock/disturbance tries to alter the magnetic topology, while the magnetization attempts to resist any modifications. We then investigate the parameter space in Section 4 to further constrain the shock parameters and the magnetization in the emission environment. In the following we will summarize the major features and illustrate their connections to observations.

We set up an emission region initially pervaded by a purely helical magnetic field with comparable poloidal and toroidal contributions. Due to the LOS effect, at the beginning the polarization is dominated by the poloidal component. A flat relativistic disturbance, traveling parallel to $B_{z}$, will then form a shock wave and propagate through the emission region. In a weakly magnetized environment $(\sigma<1)$, the magnetic field cannot compete against the shock/disturbance. Hence even a mildly relativistic shock/disturbance can permanently change the magnetic topology to a large extent (here "permanently" means on a time scale that is much longer than the flare duration). This results in drastic variations in the polarization signatures, with no signs of recovery. Such phenomena are hardly detected, thus we conclude that a weakly magnetized emission region is not favored.

In a strongly magnetized emission region $(\sigma \gtrsim 1)$, the magnetic field topology is able to survive the impact of the shock/disturbance. For the slow cases, the shock/disturbance is unable to build up a strong toroidal component, thus the polarization signatures usually exhibit rather erratic fluctuations, which are typical in observations. If the shock/disturbance is slightly faster, then it is plausible to build up the toroidal component and lead to significant polarization variations such as the PA rotations; but in both cases, the flux will not increase sufficiently to produce a flare. Such polarization variations with no obvious flares

are consistent with some observations (e.g., Itoh et al. 2013; Jorstad et al. 2013). However, 
Kirk et al. (2000) and Achterberg et al. (2001) have shown that for relativistic shocks, the hardest obtainable particle power-law index is approximately

$$
p=\frac{r_{c}+2}{r_{c}-1}
$$

where $p$ is the power-law index and $r_{c}$ is the shock compression ratio mentioned previously. We estimate that the strongest shock in our slow disturbance cases can only reach a nonthermal particle spectrum of power-law index of $\sim 4$, which is too soft to fit the general blazar SEDs. In addition, relativistic collisionless shocks in the highly magnetized regime may be unable to generate fluctuating magnetic fields and hence inhibit Fermi acceleration (e.g., Sironi et al. 2015). Therefore, a slow shock/disturbance may not efficiently accelerate nonthermal particles that are necessary to generate a flare. This poses a tricky problem for slow shocks. On the other hand, for a relatively fast shock/disturbance, the polarization variations can either fluctuate around some mean value or smoothly rotate in the PA and present a restoring phase at the end of the flare; both are detected in observations (e.g., Larionov et al. 2008; Abdo et al. 2010; Blinov et al. 2015). In summary, we prefer a strongly magnetized emission region, in which fast disturbances can be the driver of the flaring activities.

Observations and spectral variability fittings have shown that the typical Lorentz factor of the blazar emission region is within a few tens (e.g., Jorstad et al. 2005; Böttcher et al. 2013). This is likely to be the upper limit of the shock speed. Therefore, a sufficiently magnetized emission environment will mostly prohibit any dramatic polarization variations, such as the PA rotations and the sudden changes in the PD (flares in the polarized flux). For the blazar emission regions with a weaker magnetization, the PD flares and the PA rotations are triggered by strong shocks. Hence we expect strong nonthermal electron injections. Therefore, intense polarization variations are probably accompanied by vigorous multiwavelength flares. These findings are consistent with observations (Blinov et al. 2015). Another implication is that the blazar emission region presumably maintains a relatively high magnetization, thus it cannot dissipate too much magnetic energy during the shock-initiated flaring activities.

An alternative may weaken some of the constraints mentioned above. In a highly magnetized region, magnetic reconnection can also be the driver of flares. Several authors have illustrated that reconnection can efficiently accelerate nonthermal particles to form a powerlaw spectrum (Guo et al. 2014; Sironi \& Spitkovsky 2014; Li et al. 2015). In our simulations, no preexisting turbulent magnetic field is present; but in reality, some amount of turbulence may exist. Therefore, during the shock propagation, magnetic reconnection can happen simultaneously, especially in the compression regions at the shock front. This can provide extra nonthermal particles, thus a slow shock in a partially turbulent, partially ordered 
(such as a helical geometry) magnetic field structure may generate the necessary nonthermal electrons. Several models investigating the reconnection in a highly magnetized environment have been proposed to explain very fast blazar events (Giannios et al. 2009; Deng et al. 2015; Guo et al. 2015). Meanwhile, recent simultaneous fittings of blazar SEDs, light curves and polarization signatures also favor a reconnection process (Zhang et al. 2015; Chandra et al. 2015). During a reconnection event, the magnetic field topology can be greatly modified, which may give rise to some interesting polarization signatures as well. Therefore, we expect that even in a highly magnetized environment, if the reconnection drives the flaring activities, considerable polarization variations are possible. Future observations featuring both the radiation and polarization signatures, along with detailed modelings and simulations of the shock and magnetic reconnetion, can possibly distinguish the two mechanisms and further constrain the physics of blazar emission regions.

We thank the referee for insightful and constructive comments. HZ thanks Fan Guo, Shengtai Li and Xiaocan Li for helpful discussions. HZ, WD and HL are supported by the LANL/LDRD program and by DoE/Office of Fusion Energy Science through CMSO. MB acknowledges support by the South African Research Chairs Initiative (SARChI) of the Department of Science and Technology and the National Research Foundation 1 of South Africa. Simulations were conducted on LANL's Institutional Computing machines.

\section{REFERENCES}

Abdo, A. A., et al., 2010, Nature, 463, 919

Achterberg, A., Gallant, Y. A., Kirk, J. G., \& Guthmann, A. W. 2001, MNRAS, 328, 393

Aharonian, F. A., et al., 2007, ApJ, 664, L71

Albert, J., Aliu, E., Anderhub, H., et al. 2007, ApJ, 669, 862

Barnacka, A., Moderski, R., Behera, B., Brun, P., \& Wagner, S., 2014, A\&A, 567, A113

Blinov, D., Pavlidou, V., Papadakis, I., et al. 2015, arXiv:1505.07467

Böttcher, M., \& Dermer, C. D., 2010, ApJ, 711, 445

\footnotetext{
${ }^{1}$ Any opinion, finding and conclusion or recommendation expressed in this material is that of the authors and the NRF does not accept any liability in this regard.
} 
Böttcher, M., Reimer, A., Sweeney, K., \& Prakash, A., 2013, ApJ, 768, 54

Chandra, S., Zhang, H., Kushwaha, P., et al. 2015, arXiv:1507.06473

Chatterjee, R., Bailyn, C. D., Bonning, E. W., Buxton, M., Coppi, P., Fossati, G., Isler, J., Maraschi, L., \& Urry, C. M., 2012, ApJ, 749, 191

Chen, X., Chatterjee, R., Zhang, H., et al. 2014, MNRAS, 441, 2188

Ciprini, S., 2011, in proc. of 2011 Fermi Symposium - eConf C110509 (arXiv:1112.2639)

Deng, W., Li, H., Zhang, B., \& Li, S. 2015, ApJ, 805, 163

Dermer, C. D., et al., 1992, A\&A, 256, L27

Giannios, D., Uzdensky, D. A., \& Begelman, M. C. 2009, MNRAS, 395, L29

Graff, P. B., Georganopoulos, M., Perlman, E. S., \& Kazanas, D. 2008, ApJ, 689, 68

Guo, F., Li, H., Daughton, W. \& Liu, Y., 2014, PRL, 113, 155005

Guo, F., Liu, Y.-H., Daughton, W., \& Li, H. 2015, ApJ, 806, 167

Itoh, R., Fukazawa, Y., Tanaka, Y. T., et al. 2013, ApJ, 768, L24

Joshi, M. \& Böttcher, M., 2007, ApJ, 662, 884

Jorstad, S. G., Marscher, A. P., Lister, M. L., et al. 2005, AJ, 130, 1418

Jorstad, S. G., Marscher, A. P., Smith, P. S., et al. 2013, ApJ, 773, 147

Kiehlmann, S., Savolainen, T., Jorstad, S. G., et al. 2013, European Physical Journal Web of Conferences, 61, 06003

Kirk, J. G., Guthmann, A. W., Gallant, Y. A., \& Achterberg, A. 2000, ApJ, 542, 235

Komissarov, S. S., \& Lyutikov, M. 2011, MNRAS, 414, 2017

Laing, R. A. 1980, MNRAS, 193, 439

Laing, R. A., \& Bridle, A. H. 2014, MNRAS, 437, 3405

Larionov, V. M., Jorstad, S. G., Marscher, A. P., et al. 2008, A\&A, 492, 389

Larionov, V. M., et al., 2013, ApJ, 768, 40

Li, S., \& Li,H., 2003, Los Alamos National Lab. Tech. Rep. LA-UR-03-8935 
Li, X., Guo, F., Li, H., \& Li, G. 2015, arXiv:1505.02166

Lyutikov, M., Pariev, V. I., \& Gabuzda, D. C., 2005, MNRAS, 360, 869

Mannheim, K., \& Biermann, P. L., 1992, A\&A, 253, L21

Marscher, A. P. \& Gear, W. K., 1985, ApJ, 298, 114

Marscher, A. P., et al., 2008, Nature, 452, 966

Marscher, A. P., 2014, ApJ, 780, 87

Morozova, D. A., et al., AJ, 148, 42

Mücke, A., \& Protheroe, R.J., 2001, Astropart. Phys, 15, 121

Pushkarev, A. B., Gabuzda, D. C., Vetukhnovskaya, Yu. N., \& Yakimov, V. E., 2005, MNRAS, 356, 859

Scarpa, R., \& Falomo, R. 1997, A\&A, 325, 109

Sikora, M., et al., 1994, ApJ, 421, 153

Sironi, L., \& Spitkovsky, A. 2014, ApJ, 783, L21

Sironi, L., Keshet, U., \& Lemoine, M. 2015, arXiv:1506.02034

Sokolov, A., Marscher, A. P., \& McHardy, I. M. 2004, ApJ, 613, 725

Spada, M., Ghisellini, G., Lazzati, D., \& Celotti, A. 2001, MNRAS, 325, 1559

Spitkovsky, A. 2008, ApJ, 682, L5

Summerlin, E. J., \& Baring, M. G. 2012, ApJ, 745, 63

Tavecchio, F., Ghisellini, G., Bonnoli, G., \& Ghirlanda, G., 2010, MNRAS, 405, L94

Zhang, H., Chen, X. \& Böttcher, M., 2014, ApJ, 789, 66

Zhang, H., Chen, X., Böttcher, M., Guo, F., \& Li, H. 2015, ApJ, 804, 58

This preprint was prepared with the AAS IATEX macros v5.2. 

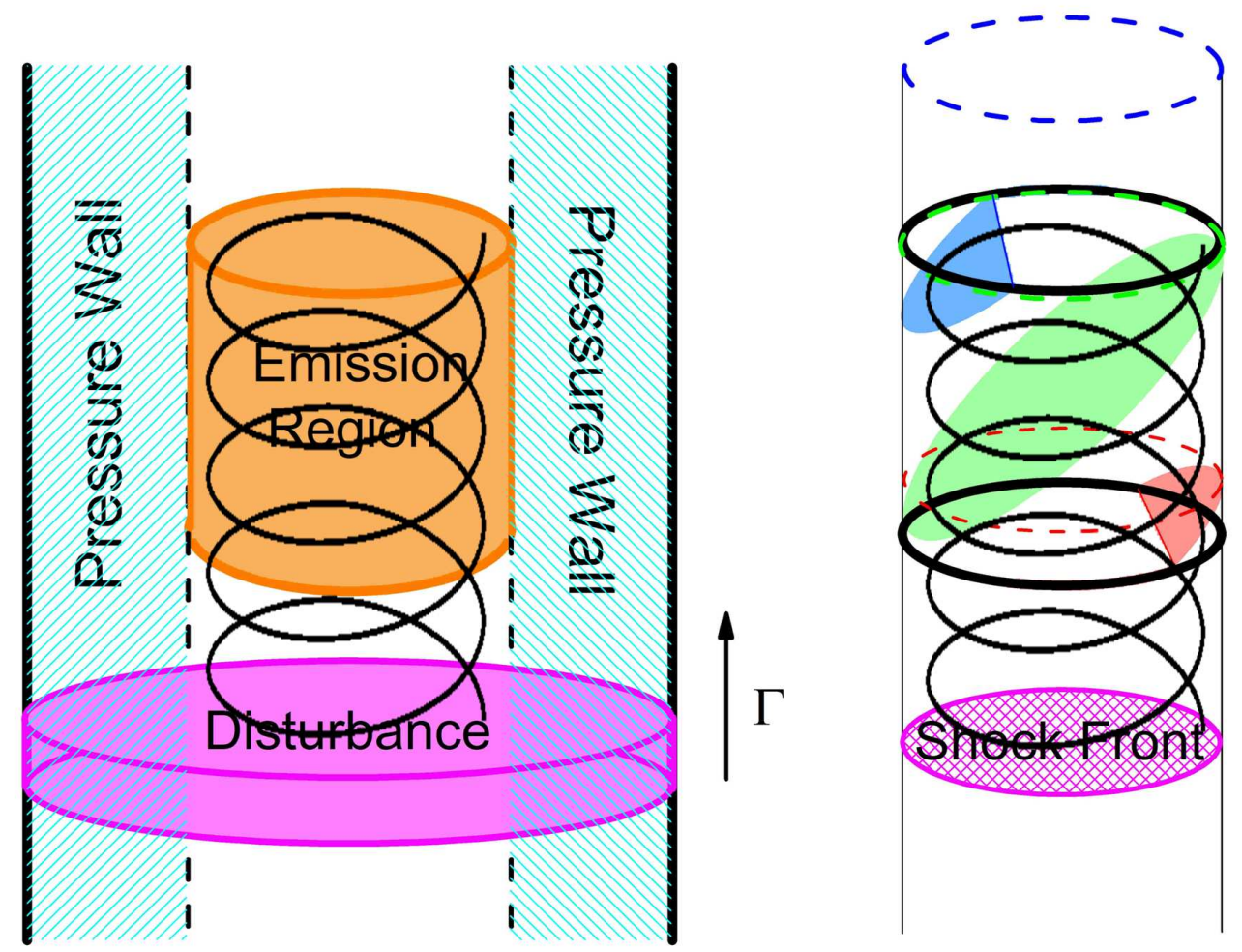

Fig. 1.- Left: a sketch of the model setup. The cylindrical simulation domain is pervaded by a helical magnetic field with exponential cutoff at the edge, which is held by a pressure wall. The emission region has a fixed height, while its radius extends to roughly the pressure wall where the magnetic field has been exponentially cut off. The disturbance is a flat region traveling upward in the simulation frame. It will form a shock wave which will propagate through the emission region and modify the local plasma conditions, and inject fresh nonthermal particles at its front. As the disturbance and the shock are relativistic, they are likely to stick together during the propagation. Right: a sketch of the LTTEs. The shock/disturbance will propagate through the emission region in the comoving frame of the emission region. The red, green and blue dash circles refer to the location of the shock front at approximately the rising, peak and declining phases of the flare, respectively; the corresponding shapes and locations of the flaring region, indicating points of equal photon arrival times at the observer, are shown by the light red, light green and light blue shapes, respectively. Notice that in our simulations, the shock front is not necessarily flat as in the sketch. 

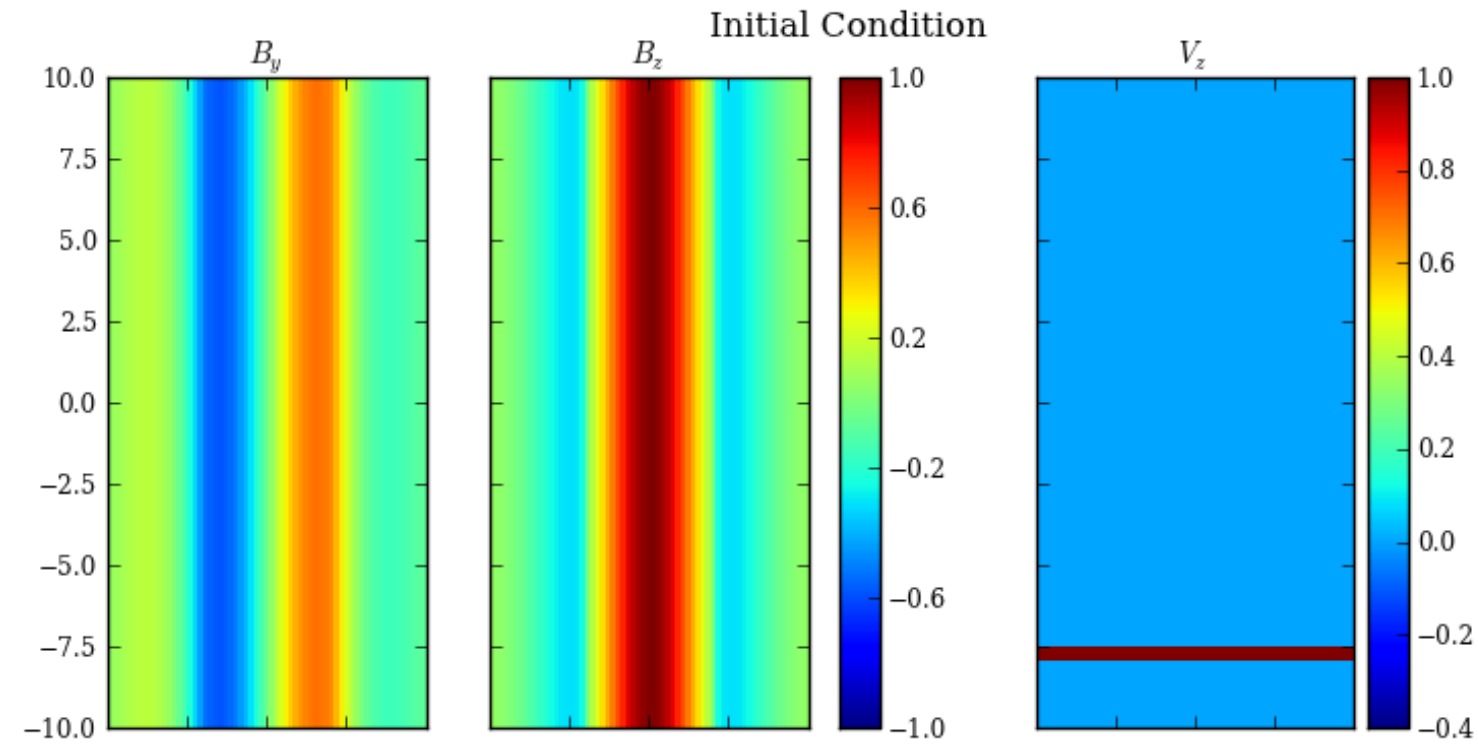

Fig. 2.- Initial condition of the RMHD simulation in the $x z$ plane. We only cut part of the simulation in $z$ direction that is related to the emission region. The emission region ranges from -2.5 to 2.5. The three panels are the toroidal component $B_{y}$, which represents the $B_{\phi}$ in this plane for a helical magnetic field ( $B_{x}$ is trivial in this plane), the poloidal component $B_{z}$ and the velocity of the disturbance $V_{z}$. All color bars show values in the RMHD code units. 

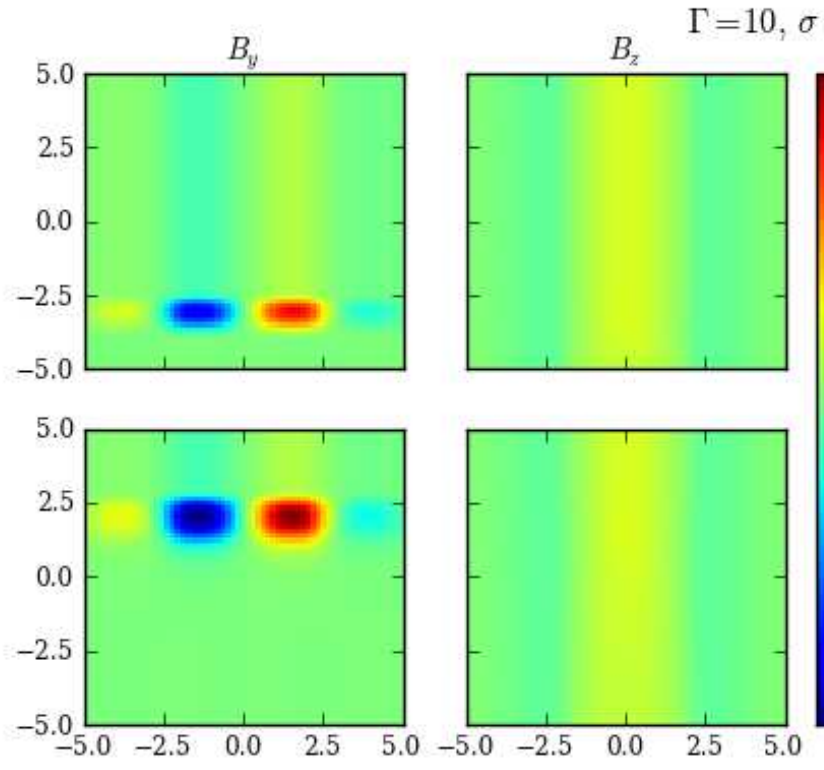
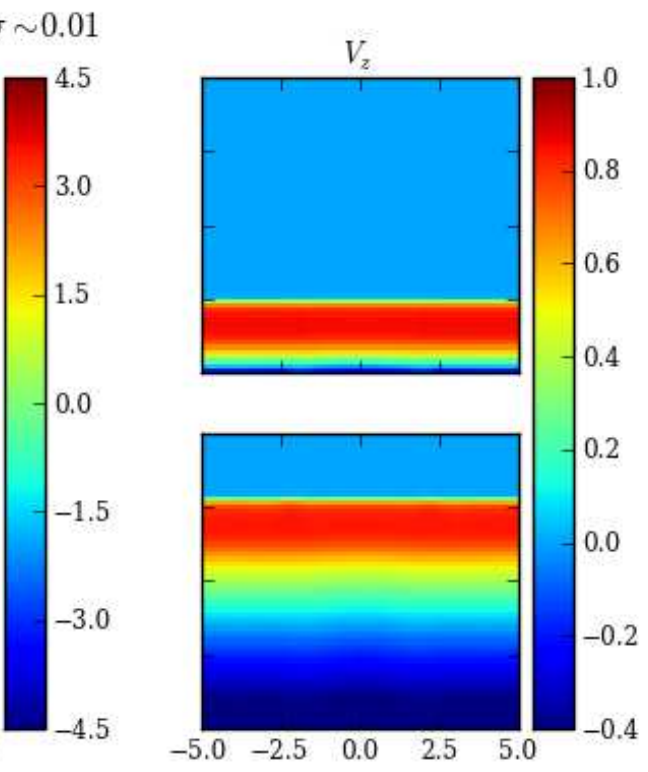

Fig. 3.- RMHD simulation results for Case I. The first row shows the magnetic field structures and the plasma velocity when the disturbance is moving into the emission region. The second row shows the same information when the disturbance is moving out. Notice the color bars for the magnetic fields are different from Fig. 2, and the region is cut smaller. Otherwise plots are as in Fig. 2.

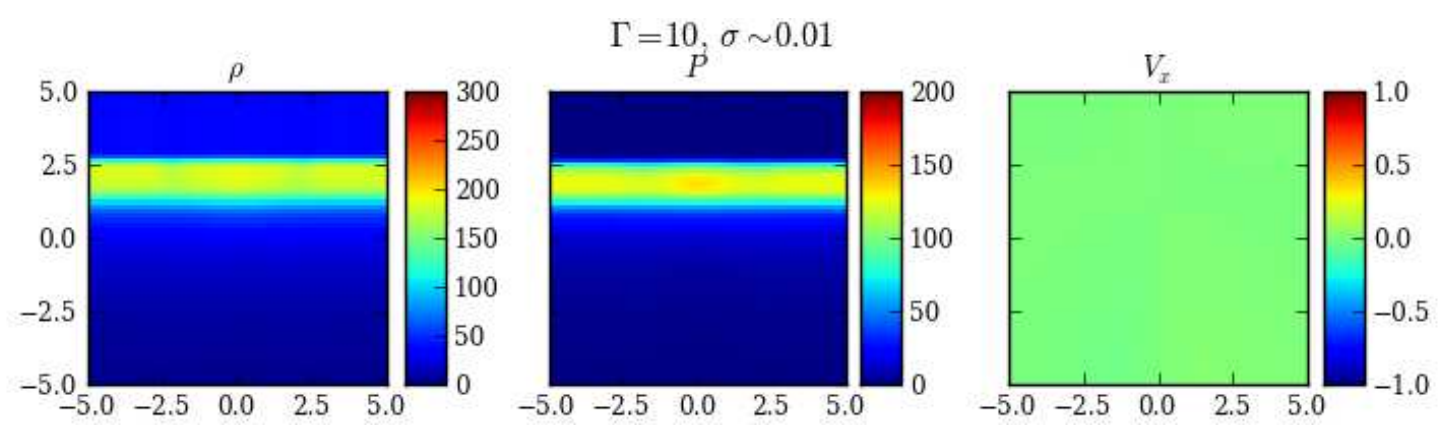

Fig. 4.- Hydro parameters in the $x z$ plane for Case I, when the disturbance is moving out of the emission region. The three panels are the plasma density $\rho$, the pressure $P$, and velocity in $x$ direction $V_{x}$, which represents the velocity in radial. All quantities are in code units. 


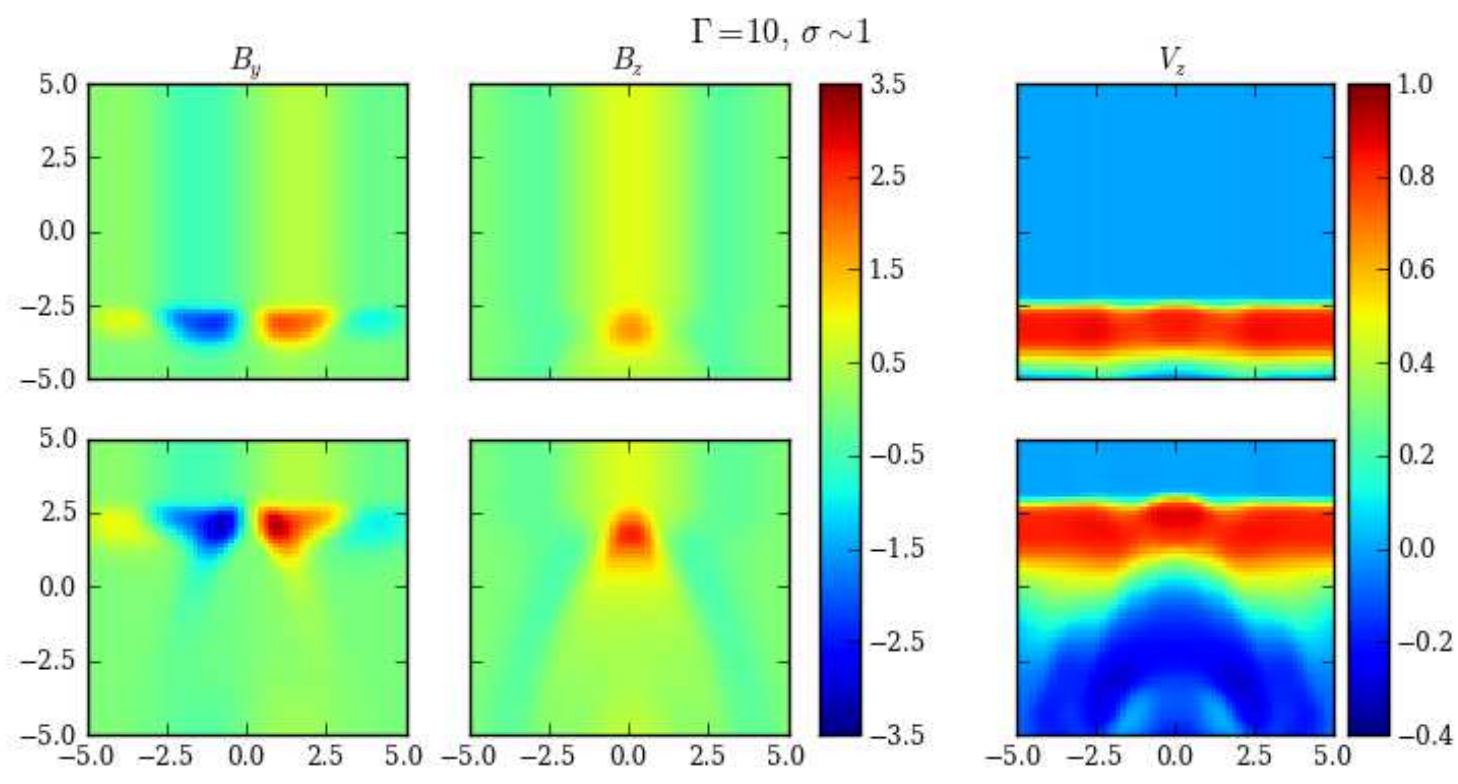

Fig. 5.- RMHD simulation results for Case II. Plots are as in Fig. 3,
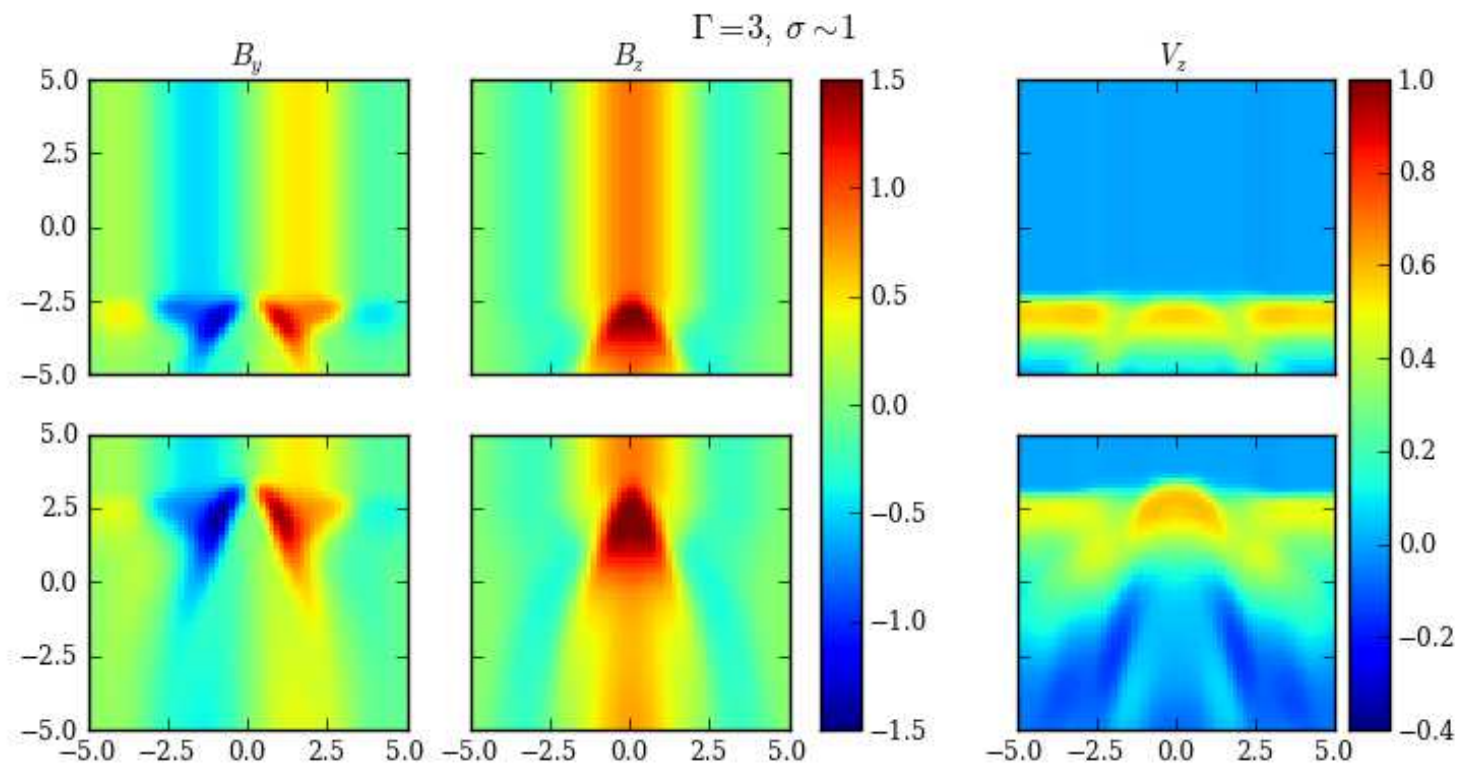

Fig. 6.- RMHD simulation results for Case III. Plots are as in Fig. 3, 


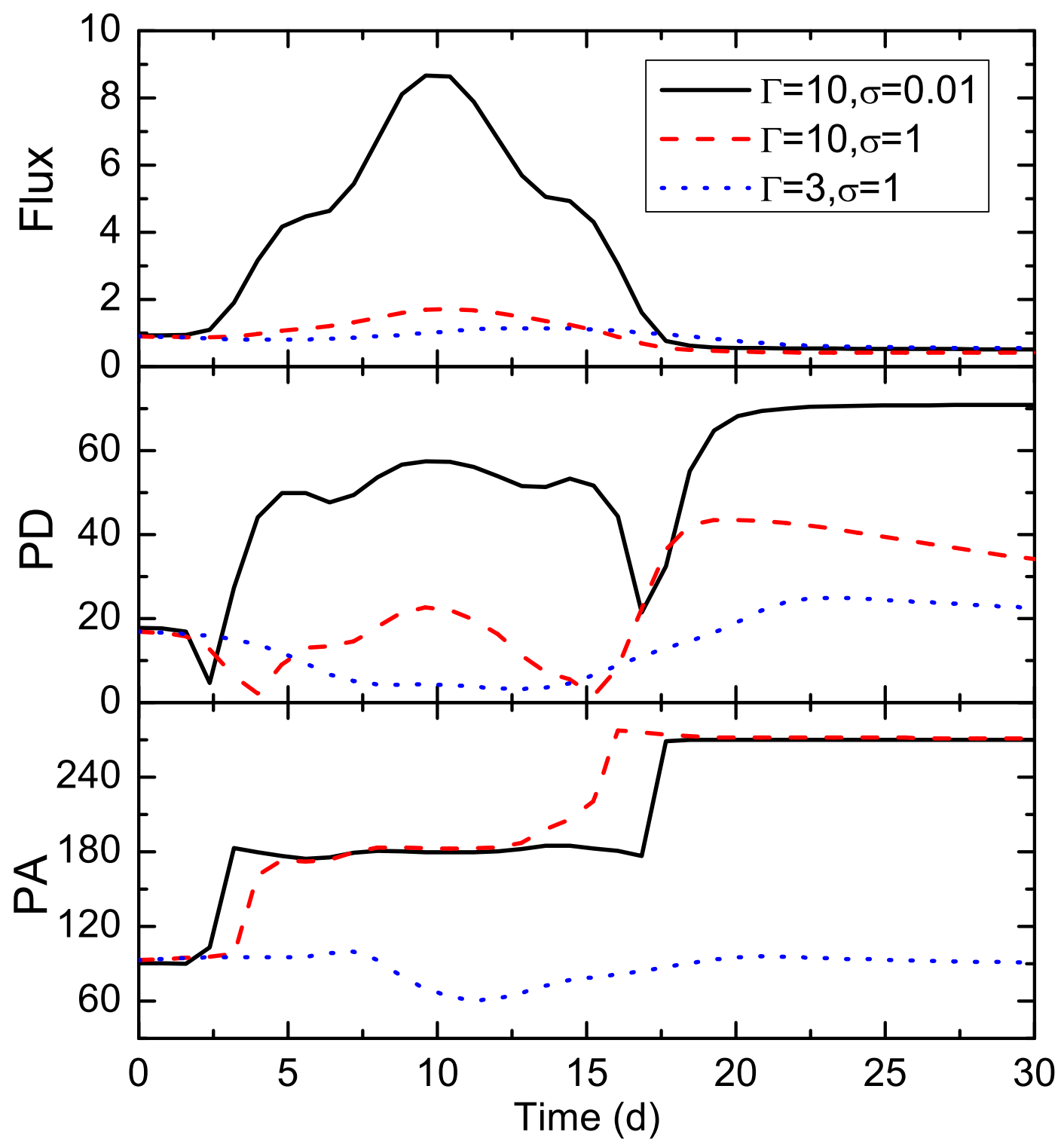

Fig. 7.- Time-dependent radiation and polarization signatures. The $\mathrm{x}$ axis is time in days. The upper panel shows the relative flux in the optical band. The middle panel is the optical $\mathrm{PD}$ in percentage. The lower panel is the corresponding PA in degree. Black solid lines are for Case I, red dash for Case II, and blue dot for Case III. 

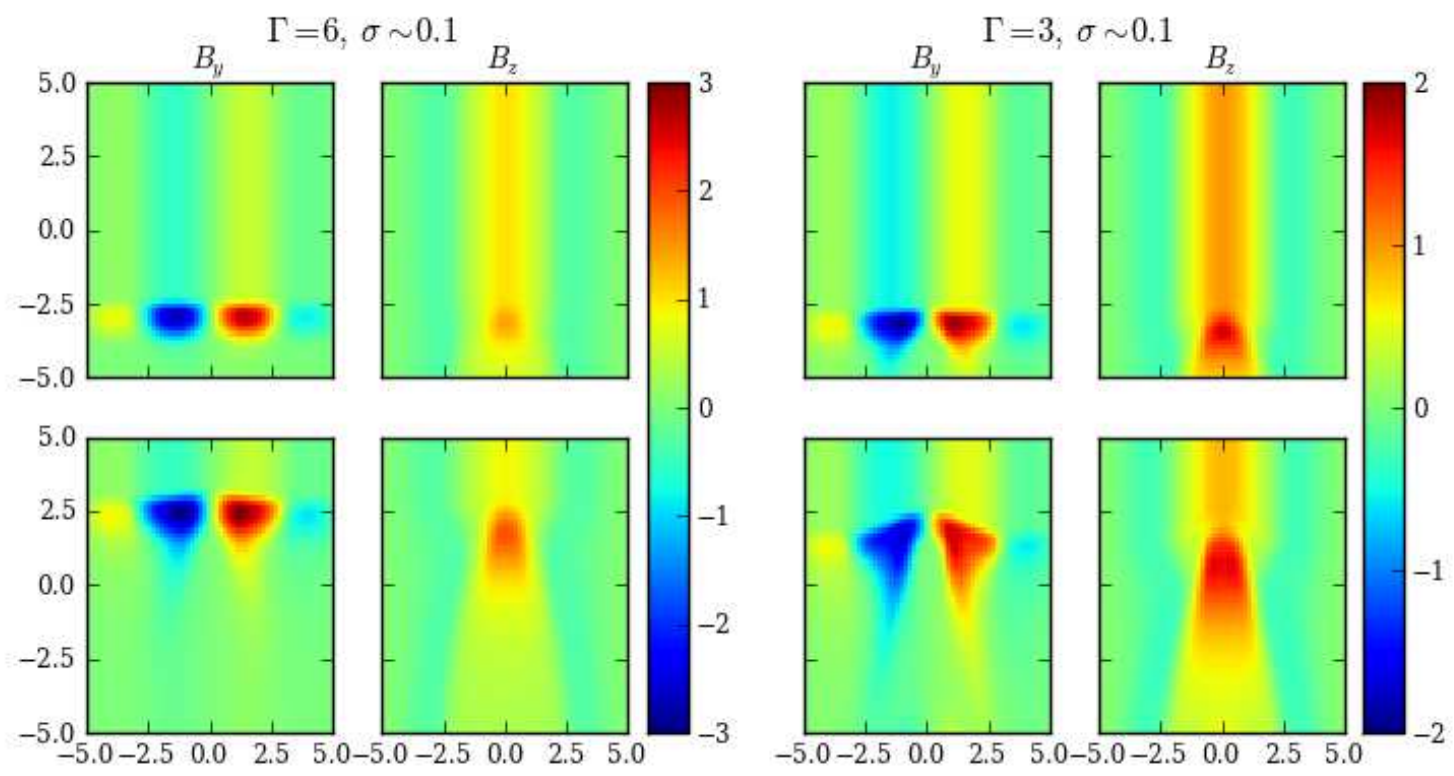

Fig. 8.- RMHD simulation results for a weakly magnetized environment $(\sigma \sim 0.1)$. We no longer plot the disturbance velocity $V_{z}$. The left panel is for a medium speed disturbance $(\Gamma=6)$, the right a slow speed disturbance $(\Gamma=3)$. Otherwise plots are as in Fig. 3 , 


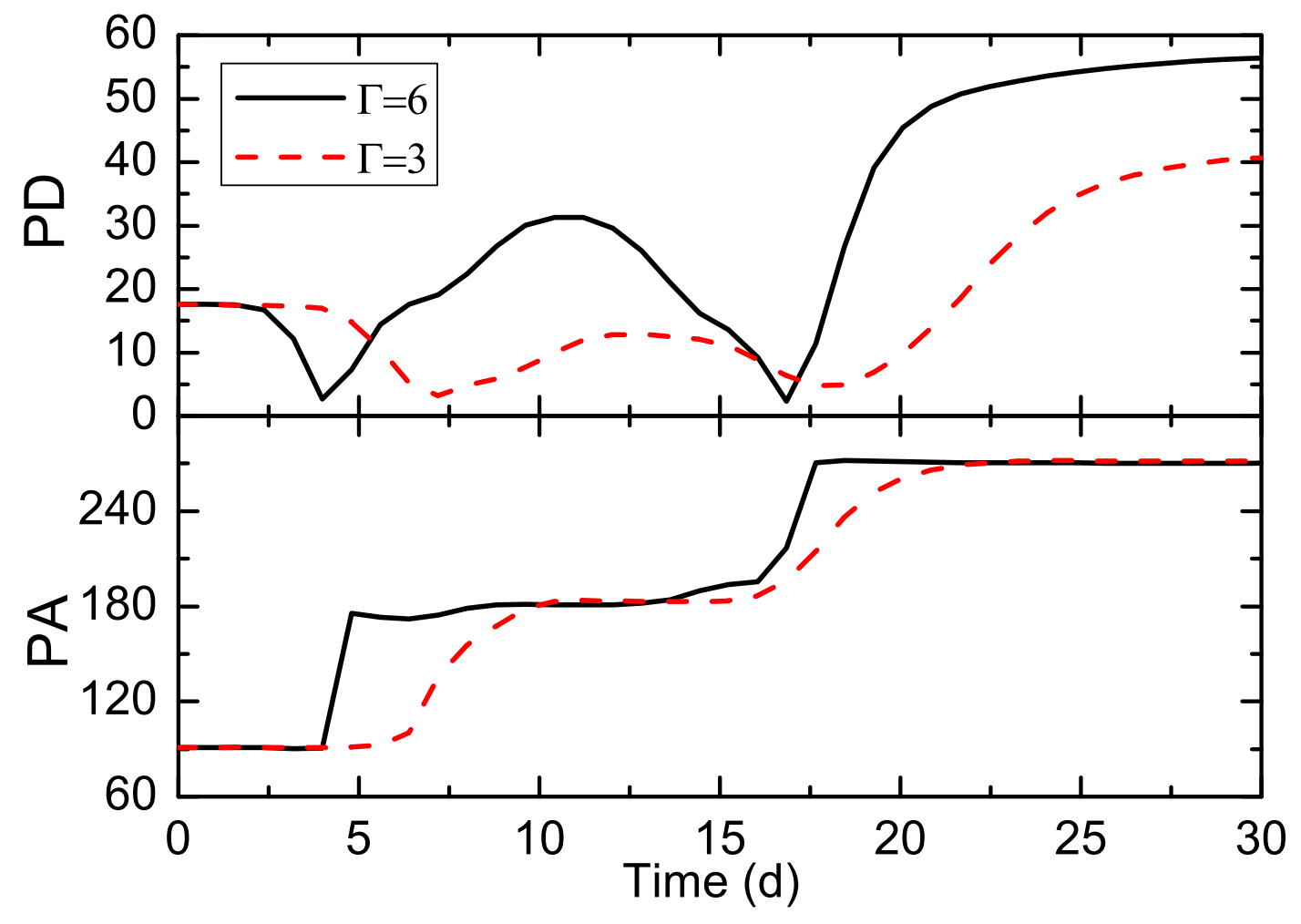

Fig. 9.- Time-dependent radiation and polarization signatures for $\sigma \sim 0.1$. The relative flux is not plotted. Black solid lines are for $\Gamma=6$, red dash are for $\Gamma=3$. Otherwise panels and curves are as in Fig. 7 . 


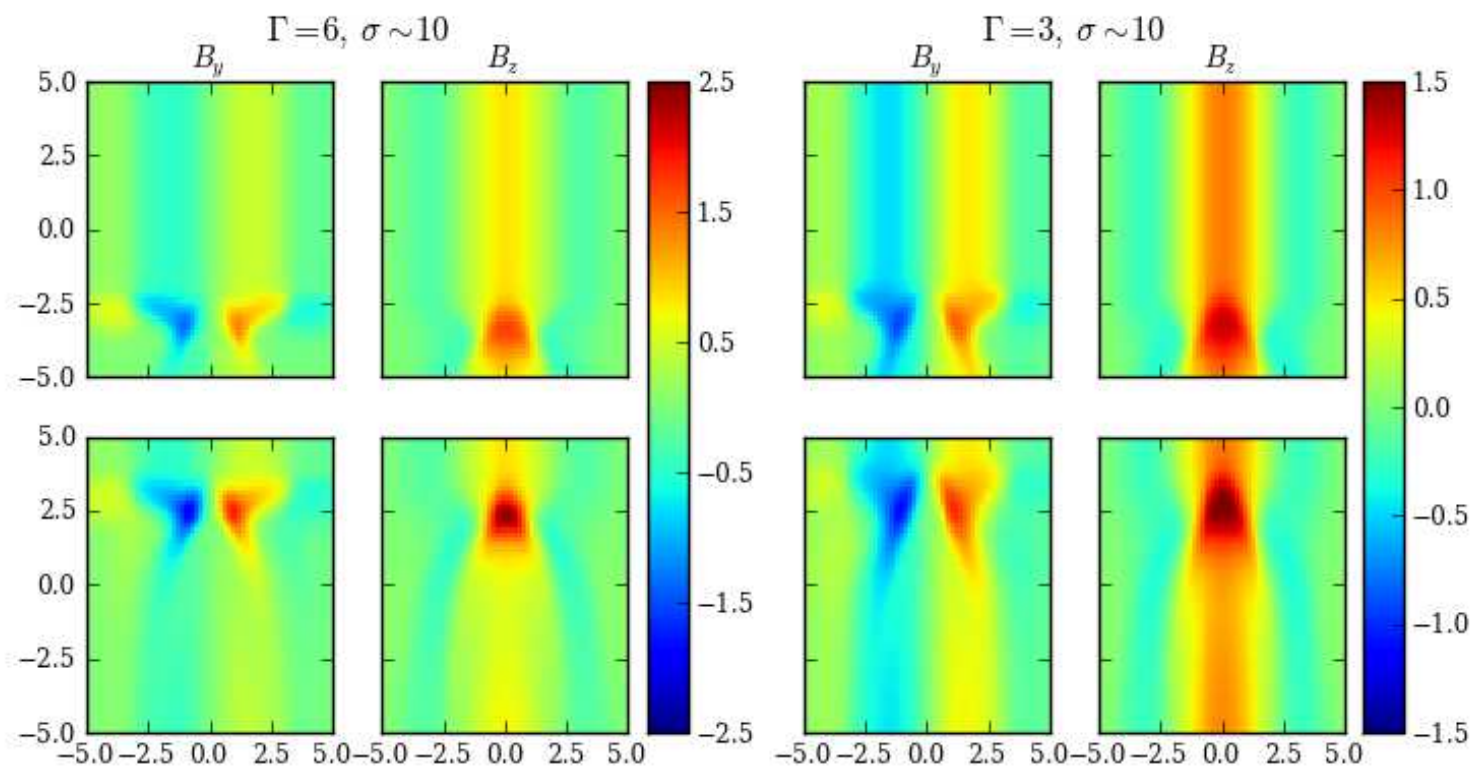

Fig. 10.- RMHD simulation results for a strongly magnetized environment $(\sigma \sim 10)$. Plots are as in Fig. 8 


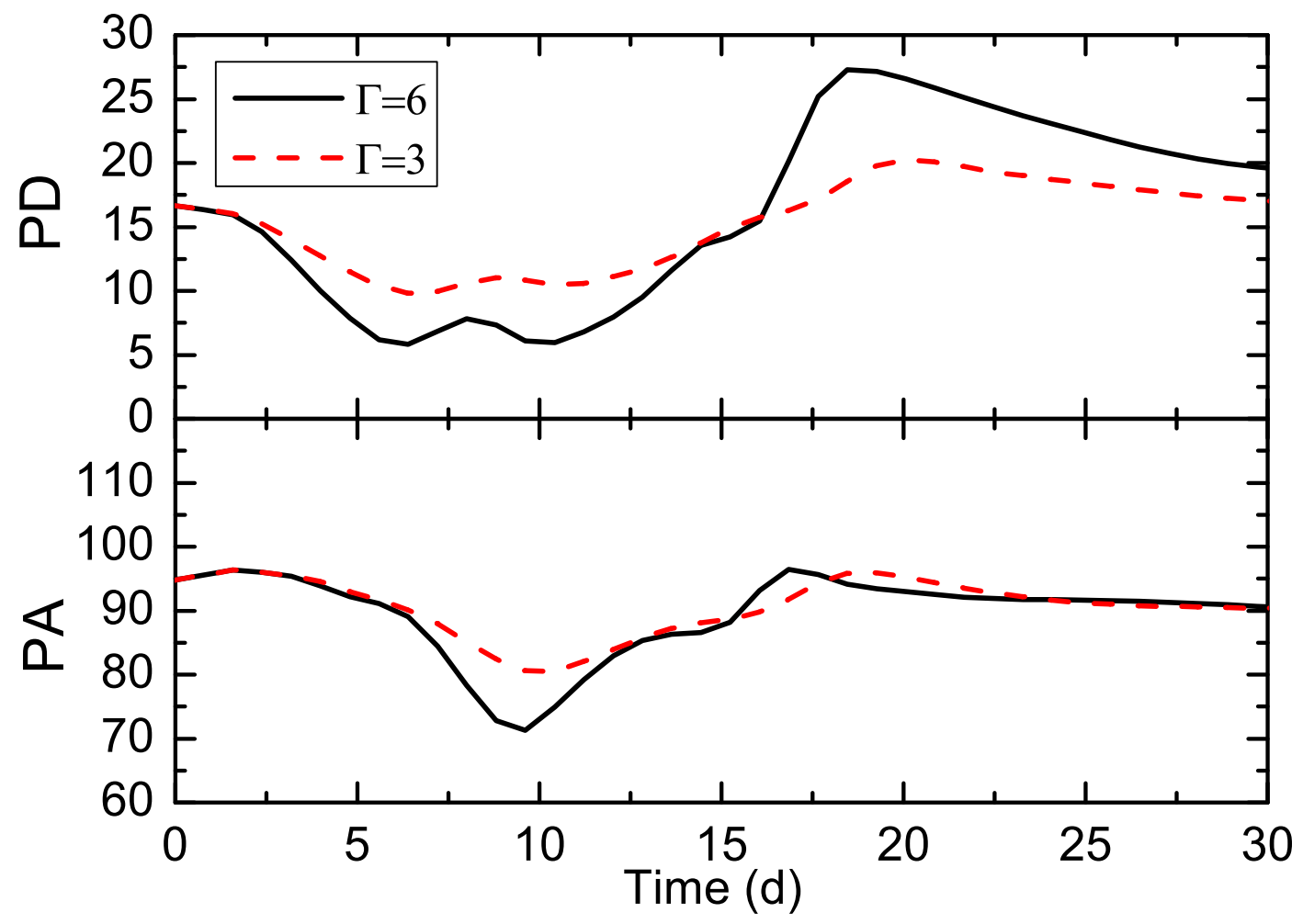

Fig. 11.- Time-dependent radiation and polarization signatures for $\sigma \sim 10$. Panels and curves are as in Fig. 9 . 

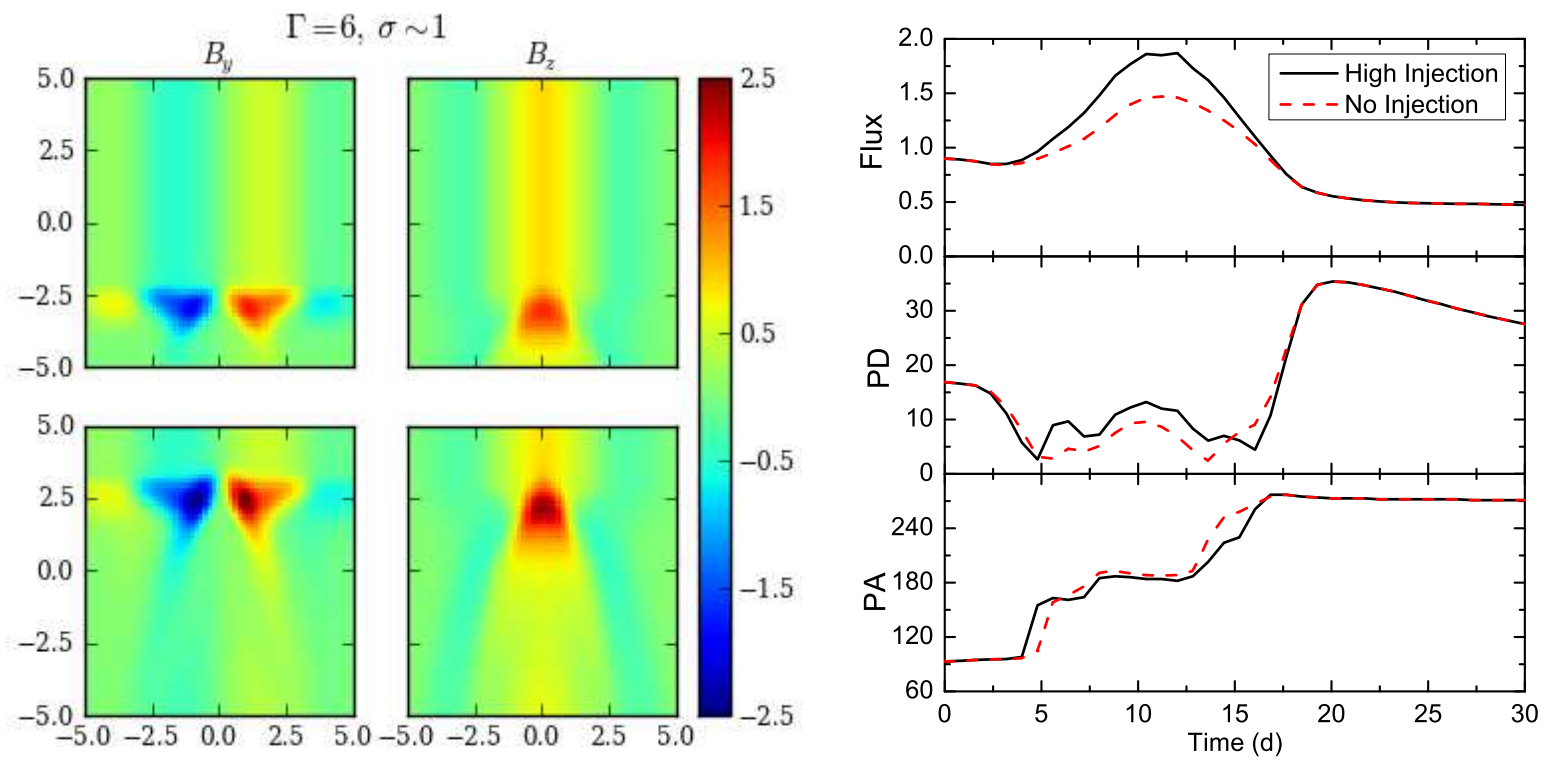

Fig. 12.- RMHD simulations and the associated time-dependent radiation and polarization signatures for $\Gamma=6, \sigma \sim 0.1$. The left side is the RMHD simulation results; plots are similar in Fig. 8. The right side is the 3DPol results. Black solid lines are for the high injection case, red dash are for the no injection case; otherwise panels and curves are as in Fig. 7. 